Review Article

\title{
The Potential Use of Melatonin for Preventing Cisplatin Ototoxicity: An Insight for a Clinical Approach
}

\author{
Juliana Gusmão de Araujo, André Luiz Lopes Sampaio, \\ Alessandra Ramos Venosa, and Carlos Augusto Costa Pires de Oliveira
}

University of Brasília, 70680-395 Brasília, DF, Brazil

Correspondence should be addressed to Juliana Gusmão de Araujo; gusmao.juliana@yahoo.com

Received 16 May 2014; Revised 15 July 2014; Accepted 24 July 2014; Published 18 August 2014

Academic Editor: Tino Just

Copyright ( 2014 Juliana Gusmão de Araujo et al. This is an open access article distributed under the Creative Commons Attribution License, which permits unrestricted use, distribution, and reproduction in any medium, provided the original work is properly cited.

\begin{abstract}
Cisplatin, one of the most effective and potent anticancer drugs, is used in the treatment of a wide variety of both pediatric and adult malignancies. However, the chemotherapeutic use of cisplatin is limited by its serious side effects, such as nephrotoxicity and ototoxicity. Ototoxicity produced by cisplatin is usually persistent, depending on the age of the patient, the cumulative number of doses, the number of chemotherapy cycles, the history of noise exposure, and deteriorating renal function. The mechanism of the ototoxicity caused by cisplatin is based on the generation of reactive oxygen species, which interfere with the antioxidant protection of the organ of Corti. Thus, protecting the cochlea with antioxidants ameliorates ototoxicity from cisplatin. In this context, melatonin appears as a therapeutic option for preventing the ototoxic effects of cisplatin, since the research in the last decade has proven its ability to be both a direct free radical scavenger and indirect antioxidant. In this sense, some of the evidence suggesting that melatonin is efficient for combating cisplatin-induced ototoxicity is summarized and discussed in this paper.
\end{abstract}

\section{Introduction}

Cisplatin is an antineoplastic drug widely used in the treatment of various types of cancer, mainly solid tumors like testicular, ovarian, lung, head, and neck cancers. Nevertheless, its collateral effects, such as nephrotoxicity, neurotoxicity, and ototoxicity, limit its use. Some of these side effects, such as ototoxicity, are dose dependent and this fact prohibits the use of higher doses, which could maximize its antineoplastic activity.

While the nephrotoxicity can be diminished or controlled with hydration therapy and diuretics, ototoxicity still remains without an effective treatment. In the last year, many drugs have been studied in an attempt to reduce the cochlear damage induced by cisplatin.

Cisplatin-induced ototoxicity is usually manifested by bilateral, progressive, and usually irreversible sensorineural hearing loss, initially occurring in the higher frequencies since cisplatin primarily damages outer hair cells from the basal turn of cochlea and the spiral ganglion neurons [1].
Although ototoxicity caused by cisplatin may occur within hours to days after drug administration, delayed ototoxicity from cisplatin may occur in children [1]. Approximately $23-54 \%$ of adults and $45 \%$ of pediatric patients with head and neck cancer treated with cisplatin develop ototoxicity [2].

There are several risk factors to cisplatin-induced hearing loss, among which are cumulative doses, age, exposure to other ototoxic drugs, cranial irradiation, depleted nutritional state, renal insufficiency, noise exposure, and preexisting hearing loss [3-5].

There have also been several publications suggesting a relationship between genes and susceptibility to cisplatin ototoxicity. Certain gene polymorphisms appear to play a role in increasing or decreasing the likelihood of ototoxicity in patients treated with cisplatin chemotherapy. Cisplatininduced hearing loss has also been studied in patients with mitochondrial mutations [6].

Many cisplatin protective agents have been evaluated in experimental and clinical studies in an effort to ameliorate cisplatin-induced ototoxicity. Some drugs may protect 
against ototoxicity; however they may also reduce the antineoplastic activity of cisplatin, which would have disastrous effects on the patients' prognosis.

In this scenario, melatonin appears as a potentially useful substance in preserving audibility. The purpose of this paper was to review articles concerning the potential effect of melatonin as a protective agent against cisplatin-induced ototoxicity. Some of the evidence suggesting that melatonin is efficient in combating cisplatin-induced ototoxicity will be summarized and discussed in this paper.

\section{Cisplatin-Induced Ototoxicity Mechanism}

Various drugs and toxins present in the circulation or the middle ear may reach the inner ear and cause a loss of auditory sensitivity. Cisplatin and some antibiotic substances cause the most important clinical form of ototoxicity.

Cell death in tumor cells is primarily through apoptosis, although tumor cells in vitro may undergo necrosis when exposed to high concentrations of cisplatin [4]. The effect of cisplatin as an antineoplastic drug is due to its cytotoxic action. It is characterized as being analogous to that of the alkylating agents. However, as opposed to classic alkylating agents, cisplatin binds to the DNA to form intrastrand crosslinks and adducts that cause changes in the conformation of the DNA and affect DNA replication [7].

Other mechanisms of cisplatin cytotoxicity include mitochondrial damage, decreased ATPase activity, and altered cellular transport mechanisms. While considered cell cycle nonspecific, cytotoxicity is increased with exposure during the S-phase. Cisplatin causes cell cycle arrest in the G2-phase and then induces programmed cell death or apoptosis [8]. Cisplatin activates nicotinamide adenine dinucleotide phosphate (NADPH) oxidase, an enzyme that produces superoxide radicals, leading to a dramatic increase in superoxide production in cochlear cell lines. Superoxide can lead to the formation of hydrogen peroxide. The latter can be catalyzed by iron to form the very reactive hydroxyl free radical [4].

Ototoxicity appears to be associated with high peak cisplatin levels and is attributed to cisplatin's ability to generate reactive oxygen species (ROS) [4]. ROS can then deplete cochlear tissues of antioxidant protective molecules, for example, glutathione and antioxidant enzymes. This may allow lipid peroxidation, as shown by increased concentrations of malondialdehyde, toxic lipid peroxides, and aldehydes. These can increase calcium influx and apoptosis in cells of the cochlea [5].

Cisplatin causes changes in the cochlear histology structure, such as the loss of the normal cytoarchitecture of the organ of Corti and reduction of neurons in the spiral ganglion due to cell alterations [9].

\section{Melatonin}

Melatonin is a remarkably diverse molecule that has been identified in species throughout the animal kingdom and, in the last decade, it has also been found in plants [10]. Melatonin is synthesized and released into the circulation and particularly into cerebrospinal fluid by the pineal gland. Immune system cells, the brain, the airway epithelium, bone marrow, ovaries, the skin, and probably other tissues also produce it. The production of melatonin increases during the period of darkness and is maintained at minimal levels during the period of light, giving rise to a circadian rhythm that is imposed by the light/dark cycle [11].

Besides its properties as a circadian rhythm transducer, several other actions for this interesting molecule have been uncovered in the last three decades. Melatonin participates in many important physiological functions, including the control of seasonal reproduction as well as influences on the immune system and numerous other biological activities $[12,13]$.

Additionally, melatonin seems to decrease toxicity and increase the efficacy of cancer chemotherapy [14]. There is evidence that melatonin anticancer activity is due to either cytostatic mechanisms or cytotoxic action which triggers the apoptotic machinery [15]. Furthermore, chemotherapyinduced cytotoxicity may be due, at least in part, to enhanced free radical generation and melatonin is one of the most potent antioxidant agents [16]. Its direct free radical scavenging activity and its regulation of gene transcription for antioxidative enzymes are of special interest in this review.

From a chemical point of view, free radicals are species containing one or more unpaired electrons. This characteristic makes them a highly reactive species that triggers chain reaction mechanisms, which may involve molecules of high biological importance, such as DNA and proteins. Most radicals that occur in vivo are, or arise from, reactive oxygen species (ROS) or reactive nitrogen species (RNS). In healthy organisms, there is a delicate balance between the production and the removal of free radicals. Under such conditions, free radicals have a beneficial effect since they are necessary for the maturation process of cellular structures, mitogenic responses, defense system, cellular signaling systems, and insulin receptor kinase activity and in the apoptosis of defective cells [16].

However, at high concentrations, free radicals can be extremely harmful to living organisms. The role of free radicals in the development of a large variety of chronic and degenerative diseases, such as in Parkinson's disease, Alzheimer's disease, multiple sclerosis, several cardiovascular diseases, rheumatoid arthritis, fetal growth restriction, preeclampsia, and cancer, is well documented [16].

Melatonin and its metabolites efficiently protect against oxidative stress through a variety of mechanisms $[16,17]$. It has the ability to scavenge a wide variety of ROS and RNS, and it has been hypothesized that its primary function in living organisms is to protect them from oxidative stress [18]. Melatonin has most of the desirable characteristics of good free radical scavengers: it is widely distributed in the body and present in adequate concentrations; it is a broad spectrum antioxidant; it is easily transported across cellular membranes; it crosses all morphophysiological barriers; it can be regenerated, after radical quenching, and its metabolites still present antioxidant activity. Finally, it has minimal toxicity $[16,19]$. 
Melatonin detoxifies a variety of free radicals and reactive oxygen intermediates and stimulates several antioxidative enzymes. Antioxidant enzymes activities and also their gene expression exhibit endogenous rhythms under normal light/ dark conditions. These changes with time suggested that these cycles might be dependent on the circadian melatonin rhythm [20].

Antioxidants, such as melatonin, have attracted a great deal of attention as potential agents for forestalling agerelated free radical-based diseases. It is already a well-known possibility that melatonin supplementation could prevent or delay the functional deterioration of the immune system that accompanies aging. Indeed, dietary supplementation of melatonin ameliorates the debilitated immune responses associated with senescence [21,22].

Based on a substantial amount of data documenting melatonin's ability to reduce neural damage in models of Alzheimer's disease, several groups have administered melatonin to individuals diagnosed with this neurodegenerative condition in an attempt to ameliorate the disease's symptoms [2326].

Melatonin has also been tested as an agent to reduce oxidative stress in adult humans subjected to cardiopulmonary bypass surgery to reduce the severity and degree of tissue damage following ischemia/reperfusion in the brain and other organs and to prevent degenerative changes in Parkinson's disease [27-29].

Melatonin has proven to be useful as a treatment for septic shock in premature newborns as well. This beneficial action of melatonin is attributed to the antioxidant properties since multiple organ failure and death in sepsis are believed to be due, at least in part, to excessive free radical generation. Its use in adults also seems justified [30].

Because of its actions as a direct free radical scavenger and indirect antioxidant, melatonin reduces the toxicity and increases the efficacy of a large number of drugs whose side effects are well documented. Reiter et al., in 2002, summarize the beneficial effects of melatonin when combined with the following drugs: doxorubicin, cisplatin, epirubicin, cytarabine, bleomycin, gentamicin, ciclosporin, indometacin, acetylsalicylic acid, ranitidine, omeprazole, isoniazid, iron and erythropoietin, phenobarbital, carbamazepine, haloperidol, caposide-50, morphine, cyclophosphamide, and Lcysteine [12].

A variety of otoprotective compounds have been successfully used in experimental animals. The ideal protective agent, if given systemically, should have three characteristics: it should be nontoxic, it should achieve sufficiently high concentrations in the inner ear, and it must not interfere with the antitumor effect of cisplatin. Considering the low toxicity of melatonin and its ability to reduce the side effects and increase the efficacy of the drugs, its use as a combination therapy with cisplatin seems important and worthy of pursuit.

3.1. Oncostatic Properties of Melatonin. The therapeutic potential of melatonin goes beyond the treatment of jet lag or sleep disorders. It has been shown that it has antitumoral properties in many experimental conditions. A large number of studies have demonstrated that melatonin has important oncostatic properties since it inhibits cell proliferation in several cancer cell lines and additionally reduces the toxicity of antineoplastic drugs.

Melatonin has been described to reduce not only the incidence but also the cancer cell growth. Melatonin inhibition of cell growth, invasiveness, and metastatic properties in tumoral cell cultures, mainly endocrine tumours, has been reported. Additionally, synergy between melatonin and several chemotherapeutic drugs in the induction of apoptosis has also been published [31,32]. The exact mechanism by which melatonin exerts its antitumoral actions is not fully understood.

Melatonin acts as a regulator for neoplastic cell growth, particularly on tumors corresponding to the mammary gland. Experimental manipulations activating the pineal gland, or the administration of melatonin, reduce the incidence and growth rate of chemically induced mammary tumors in rodents, while pinealectomy or situations that implicate a reduction of melatonin production usually stimulate mammary carcinogenesis [33].

The antiproliferative actions of melatonin on human breast cancer cells in vitro have been confirmed by numerous laboratories and have also been seen in other tumor types, including prostate cancer, ovarian cancer, endometrial cancer, liver cancer, and osteosarcomas [34]. Physiologic concentrations of melatonin, corresponding to peak daytime and peak night-time serum values in humans, can significantly suppress the proliferation of both the estrogen receptor alphapositive and the negative human breast cancer cells in vitro $[35,36]$.

Concomitant administration of melatonin significantly reduces the frequency of thrombocytopenia, neurotoxicity, cardiotoxicity, stomatitis, and asthenia in chemotherapy with cisplatin for lung cancer $[14,37]$. The possibility of improvement in the efficacy of chemotherapy is already known in terms of both survival and quality of life through the concomitant administration of melatonin in a considerable number of patients with metastatic non-small-cell lung cancer and for a long follow-up period [38].

Melatonin has cytotoxic activity against several hematological cancer cell lines. Hematological malignancies frequently show defective apoptosis and downregulation of death receptors. Melatonin's cytotoxic effects are attributed to the activation of the extrinsic pathway of apoptosis. Melatonin also induces cell death in hematological cancer cells obtained from the peripheral blood and bone marrow of patients with acute lymphoid leukemia $[39,40]$.

Melatonin, besides its cell growth inhibition and cell cycle perturbation, triggers apoptosis in lymphoblastic cells via a specific effect on mitochondrial membrane potential, leading to cytochrome $\mathrm{c}$ release and caspase- 3 activation and playing an important role as a potential therapeutic drug in lymphoproliferative diseases [41].

Additionally, melatonin reduces the growth of the human myeloid leukemia cells HL-60, inhibiting progression from G1 to the $S$ phase of the cell cycle and increasing apoptotic cell death. Furthermore, melatonin treatment elevates 
cytochrome $\mathrm{c}$ release from the mitochondria and augments caspase-3 and caspase- 9 activities [42].

Neuroblastoma, a pediatric cancer, which is most common before the age of 10 , has its total number of cells reduced with increasing concentrations of melatonin [43]. Glioblastoma, the most common primary brain tumor, is a highly aggressive malignancy considered to be one of the most lethal cancers. The combination of chemotherapy and melatonin appears as a new approach in glioblastoma treatment [44-46].

Melatonin has been shown to induce cytotoxicity in the Ewing sarcoma cell line. Ewing sarcoma is the second most frequent type of bone cancer and affects mainly adolescents, who have a $50 \%$ survival rate 5 years after diagnosis. Current treatments include a combination of surgery, radiotherapy, and chemotherapy, which present potential serious side effects. Melatonin creates a synergy in the antitumor effect when combined with chemotherapeutic drugs. This synergism is due to the potentiation of cell death and there is a significant increase in the activation of both intrinsic and extrinsic apoptotic pathways when melatonin is combined with the treatment $[47,48]$.

3.2. Action against the Side Effects of Cisplatin. Reversible and irreversible side effects of melatonin including nephrotoxicity, bone marrow toxicity, gastrointestinal toxicity, neurotoxicity, and ototoxicity may limit its utility and therapeutic profile. Melatonin may play an important role in the prevention of these side effects.

Several antioxidant agents have been reported to either ameliorate or prevent the nephrotoxicity of cisplatin including melatonin, selenium, vitamins $\mathrm{E}$ and $\mathrm{C}, \mathrm{N}$-acetylcysteine, and many others [49]. Melatonin protects against oxidative damage associated with cisplatin in rat kidneys. The mechanism of cisplatin-induced nephrotoxicity has been postulated as follows: when cisplatin accumulates in the kidney, ROS are generated and cells undergo oxidant stress, resulting in cytotoxicity and leading to cell death [17].

Depletion of renal glutathione and glutathione peroxidase, as well as an increase in lipid peroxidation, has been observed as a result of cisplatin administration. The melatonin cotreatment restores the activity of the antioxidant enzyme glutathione peroxidase [49-51].

Serum creatinine and urea nitrogen significantly increase following cisplatin administration in rats, and these values decrease significantly with melatonin cotreatment [52]. Histological analysis shows that cisplatin causes damage in the proximal tubular cells in the kidneys of cisplatin-treated rats and these changes are also reversed by melatonin cotreatment [52]. It has been suggested that melatonin administration, even after cisplatin injection, still has protective effects, unlike other antioxidants [53].

Cisplatin chemotherapy has been reported to cause a variety of vascular side effects. There are reports of venous thromboembolic events, cerebrovascular and cardiovascular arterial occlusion in patients treated with cisplatin. Phenylephrine and $\mathrm{KCl}$ produce concentration-dependent contractions of the rat aorta. Treatment with cisplatin increased the sensitivity to phenylephrine and $\mathrm{KCl}$. Melatonin cotreatment decreased the sensitivity to both contractile agents [54].

Another benefit of melatonin cotherapy that has already been demonstrated is protection against testicular damage. Oxidative stress caused by free radicals plays an important role in the development of cisplatin-induced testicular damage, and melatonin has a potent protective effect against the testicular toxicity of this agent [55]. Cisplatin treatment causes a decrease in the epididymal sperm concentration and in the sperm motility. Treatment with melatonin provided moderate normalization of sperm concentration and full recovery of sperm motility [56]. Thus melatonin may play a role as a potential therapeutic agent for impairment of testicular function that is induced by cytotoxic chemotherapies [55].

3.3. Melatonin Effect against Other Drugs Ototoxicity. Ototoxicity caused by gentamicin and tobramycin is ameliorated by melatonin and the pineal hormone does not interfere with their antibiotic capacity $[57,58]$. This discovery supports the hypothesis that melatonin has a role in ototoxicity prevention related to the protective effect in the outer hair cells of the organ of Corti.

Gentamicin induces loss of utricular hair cells in rats, and this effect is significantly attenuated by coadministration of melatonin. Melatonin reduced reactive oxygen species production and caspase- 3 activation in the gentamicin treated utricular hair cells [59]. Guinea pigs receiving melatonin cotherapy have hearing loss significantly attenuated and their cochleae show lower rates of outer hair cell loss compared with animals treated exclusively with gentamicin [19].

It has been shown that melatonin administrated locally through catheters placed in the round window membrane niches of cochlea or through pieces of gelfoam placed in the round window membrane niches has otoprotective properties that could be beneficial in the treatment of gentamicininduced hearing loss. The development of local long-term delivery techniques to the cochlea could be an alternative in terms of reducing the levels of drugs required for effective treatment, decreasing or eliminating side effects, and avoiding alteration of drugs by liver metabolism, thereby assuring that the desired concentration of a drug is achieved only in the target area, that is, the perilymph within the scala tympani [60].

Low-dose melatonin administration also protects the inner ear from amikacin-induced ototoxicity. However, high dose melatonin facilitates its ototoxicity, possibly via the vasodilator effect, leading to an increased accumulation of amikacin in the inner ear [61].

3.4. Antioxidants Acting against Cisplatin-Induced Ototoxicity. It has been demonstrated that plasma concentrations of various antioxidants significantly decreased during cisplatinbased chemotherapy in cancer patients. It is well known that, in hearing loss associated with cisplatin, free oxygen radicals are produced in the outer hair cells, spiral ganglion stria vascularis, and in the cochlea. Several antioxidant substances have revealed protective effects against cisplatin 
ototoxicity in animal models and in human studies. The exogenous application of antioxidant molecules has become the primary focus in the treatment strategies of cisplatin-induced ototoxicity.

Vitamin C, vitamin E, and L-carnitine are important natural antioxidants, which are synergistically able to interfere with the harmful reactions initiated by free radicals. They were found to make a significant contribution to decreasing or preventing cisplatin-induced ototoxicity in rats $[62,63]$.

Resveratrol, a natural polyphenolic nonflavonoid antioxidant, has been shown to have significant anti-inflammatory, antioxidant, and immunomodulatory properties. Its antioxidant properties mainly depend on the upregulation of endogenous cellular antioxidant systems, but the compound also shows direct ROS-scavenging properties. Cisplatin-related ototoxicity has been greatly prevented by resveratrol use [64, 65].

Ginkgo biloba extract (EGB761) shows potential otoprotector effect because of its antioxidant properties [66]. This protective action was also revealed in several studies [6770]. The association of ginkgo biloba extract with cilostazol may have a synergistic effect by strongly protecting hearing and vestibular dysfunction induced by cisplatin by inhibiting reactive oxygen species production, mitochondrial pathways, and the p-extracellular regulated kinase pathway without interfering with cisplatin pharmacokinetics [71].

Edaravone, another free radical scavenger, can interact with both peroxyl and hydroxyl radicals to form oxidized compounds and it attenuates cisplatin-induced hair cell damage and ultrastructural changes in zebrafish by reducing free radicals [72]. Systemic administration of thiamine pyrophosphate yielded statistically significant protection from cisplatin toxicity in the cochlea of guinea pigs. It may also be a potential drug candidate for humans [73].

D-methionine, a sulfur-containing compound, either administered systemically or through the round window membrane, has been shown to protect from cisplatin-induced ototoxicity. Further, D-methionine does not interfere with cisplatin's antitumor action [74]. D-methionine has been studied extensively in the prevention of cisplatin-induced hearing loss $[75,76]$.

$\mathrm{N}$-acetylcysteine, a precursor to glutathione, is an antioxidant that limits the extent of the oxidative stress damage to the cell and is able to improve the oxidant/antioxidant cellular balance. $\mathrm{N}$-acetylcysteine may protect the inner ear from the ototoxic effects of cisplatin both by acting as a free radical scavenger and by inducing the production of endogenous antioxidants [77].

Rosmarinic acid has been reported to have antioxidative, anti-inflammatory, and antidepressive activities. It inhibited cisplatin-induced apoptosis via inhibition of ROS generation and other mechanisms. In this respect, it may be attributed to a decrease in the ototoxic side effect of cisplatin in humans [78].

It is known that, at times, high doses of these drugs are required to achieve the desired effects against cisplatin ototoxicity, and their systemic application may result in decreased efficacy of cisplatin's tumoricidal properties, among other collateral effects. Therefore, intratympanic administration of drugs is a method of locally treating inner ear disorders by allowing diffusion across the round window into the inner ear. This method allows higher concentration of drugs within the inner ear compared with oral or parenteral routes.

Vitamin C, a powerful antioxidant, reduces the cytotoxic effects of antineoplastic drugs. However, high doses of vitamin $\mathrm{C}$ are required to achieve the desired effects, which is substantially higher than the maximum dose a person can take daily. Intratympanic application of vitamin $\mathrm{C}$ protects against cisplatin-induced ototoxicity. In addition, intratympanic application of vitamin $\mathrm{C}$ does not produce a toxic effect on the cochlea, suggesting that the intratympanic method might be a safe drug delivery route for vitamin C [79].

Vitamin E, a slow-acting free radical scavenger, has a significant protective effect on hearing functions which are damaged by cisplatin in rats. Vitamin E may minimize inner ear damage induced by cisplatin. Intratympanic application is safer and easier and no significant adverse effects in hearing are seen [80]. Intratympanic infusions of $\mathrm{N}$-acetylcysteine may offer significant protection against cisplatin-induced inner ear toxicity [77].

Intratympanic administration of dexamethasone also reduces ototoxicity from systemic cisplatin. It reduces the formation of free radicals and protects hearing in animal models. Intratympanic dexamethasone administration is a safe, easy, and efficient way to protect from cisplatin ototoxicity, especially when administered one hour before cisplatin treatment [80-83].

Despite the fact that several drugs had been used in an attempt to prevent cisplatin-ototoxicity, melatonin seems to have a potential advantage, because it is known to be nontoxic, it achieves sufficiently high concentrations in the inner ear, and it apparently does not impair the antitumor effect of cisplatin.

3.5. Potential Use of Melatonin. There is a great deal of theoretical support for melatonin use against cisplatin-induced ototoxicity. Moreover, several studies have already found that melatonin could be effective in reducing the nephrotoxicity of cisplatin and the ototoxicity of some antibiotics, such as gentamicin and amikacin.

Lopez-Gonzalez et al., in 2000, studied the ototoxic effect of cisplatin in rats. Besides cisplantin, melatonin and a mixture of antioxidants consisting of alpha-tocopherol succinate, ascorbic acid, glutathione and $\mathrm{N}$-acetylcysteine were administered. Since the mechanism of the ototoxicity caused by cisplatin is based on the generation of reactive oxygen species, which interfere with the antioxidant protection of the organ of Corti, they hypothesized that the protection of the cochlea with antioxidants ameliorates the ototoxicity from cisplatin. The distortion product otoacoustic emissions were determined for a prolonged period of time for each animal. The ototoxicity produced by cisplatin was maximal from day 7 to day 10 after treatment, returning to normal values in a month. When melatonin and the antioxidant mixture were present, the recovery time was between 10 and 15 days after treatment, independent of the means of administration of the 
pineal product. They conclude that the ototoxicity caused by cisplatin is ameliorated by melatonin and other antioxidants [11].

\section{Conclusion}

Thus, melatonin appears to have a potential effect against cisplatin-induced ototoxicity. There is ample justification for the assumption that melatonin may be capable of overcoming hearing loss due to cisplatin treatment. Nevertheless, further studies are necessary to clarify the specific role of melatonin as a protective substance in the auditory system and certify that melatonin does not impair the antineoplastic effect of cisplatin.

\section{Conflict of Interests}

The authors declare that there is no conflict of interests regarding the publication of this paper.

\section{References}

[1] L. P. Rybak, D. Mukherjea, S. Jajoo, and V. Ramkumar, "Cisplatin ototoxicity and protection: clinical and experimental studies," Tohoku Journal of Experimental Medicine, vol. 219, no. 3, pp. 177-186, 2009.

[2] R. Ravi, S. M. Somani, and L. P. Rybak, "Mechanism of cisplatin ototoxicity: antioxidant system," Pharmacology \& Toxicology, vol. 76, no. 6, pp. 386-394, 1995.

[3] C. Bokemeyer, C. C. Berger, J. T. Hartmann et al., "Analysis of risk factors for cisplatin-induced ototoxicity in patients with testicular cancer," British Journal of Cancer, vol. 77, no. 8, pp. 1355-1362, 1998.

[4] L. P. Rybak, "Mechanisms of cisplatin ototoxicity and progress in otoprotection," Current Opinion in Otolaryngology and Head and Neck Surgery, vol. 15, no. 5, pp. 364-369, 2007.

[5] L. P. Rybak, K. Husain, C. Morris, C. Whitworth, and S. Somani, "Effect of protective agents against cisplatin ototoxicity," The American Journal of Otology, vol. 21, no. 4, pp. 513-520, 2000.

[6] L. Riedemann, C. Lanvers, D. Deuster et al., "Megalin genetic polymorphisms and individual sensitivity to the ototoxic effect of cisplatin," Pharmacogenomics Journal, vol. 8, no. 1, pp. 23-28, 2008.

[7] H.-J. Kim, G.-S. Oh, J.-H. Lee et al., "Cisplatin ototoxicity involves cytokines and STAT6 signaling network," Cell Research, vol. 21, no. 6, pp. 944-956, 2011.

[8] A. Ekborn, Cisplatin-induced ototoxicity. Pharmacokinetics, prediction and prevention, 2003.

[9] C. M. Franceschi de, T. Tochetto, A. F. Silveira Da, M. R. Fantinel, and T. D. Algarve, "Cisplatin effects on guinea pigs: cochlear histology and genotoxicity," Brazilian Journal of Otorhinolaryngology, vol. 77, no. 6, pp. 728-735, 2011.

[10] R. J. Reiter, D. X. Tan, L. C. Manchester, M. Pilar Terron, L. J. Flores, and S. Koppisepi, "Medical implications of melatonin: receptor-mediated and receptor-independent actions," Advances in Medical Sciences, vol. 52, pp. 11-28, 2007.

[11] M. A. Lopez-Gonzalez, J. M. Guerrero, F. Rojas, and F. Delgado, "Ototoxicity caused by cisplatin is ameliorated by melatonin and other antioxidants," Journal of Pineal Research, vol. 28, no. 2, pp. 73-80, 2000.
[12] R. J. Reiter, D. Tan, R. M. Sainz, J. C. Mayo, and S. Lopez-Burillo, "Melatonin: reducing the toxicity and increasing the efficacy of drugs," Journal of Pharmacy and Pharmacology, vol. 54, no. 10, pp. 1299-1321, 2002.

[13] J.-H. Kim, S.-J. Jeong, B. Kim, S.-M. Yun, D. Y. Choi, and S.H. Kim, "Melatonin synergistically enhances cisplatin-induced apoptosis via the dephosphorylation of ERK/p90 ribosomal S6 kinase/heat shock protein 27 in SK-OV-3 cells," Journal of Pineal Research, vol. 52, no. 2, pp. 244-252, 2014.

[14] P. Lissoni, S. Barni, M. Mandalà et al., "Decreased toxicity and increased efficacy of cancer chemotherapy using the pineal hormone melatonin in metastatic solid tumour patients with poor clinical status," European Journal of Cancer, vol. 35, no. 12, pp. 1688-1692, 1999.

[15] A. Gonzalez, A. del Castillo-Vaquero, A. Miro-Moran, J. A. Tapia, and G. M. Salido, "Melatonin reduces pancreatic tumor cell viability by altering mitochondrial physiology," Journal of Pineal Research, vol. 50, no. 3, pp. 250-260, 2011.

[16] A. Galano, D. X. Tan, and R. J. Reiter, "Melatonin as a natural ally against oxidative stress: a physicochemical examination," Journal of Pineal Research, vol. 51, no. 1, pp. 1-16, 2011.

[17] J. Fukutomi, A. Fukuda, S. Fukuda, M. Hara, A. Terada, and M. Yoshida, "Scavenging activity of indole compounds against cisplatin-induced reactive oxygen species," Life Sciences, vol. 80, no. 3, pp. 254-257, 2006.

[18] D.-X. Tan, L. C. Manchester, M. P. Terron, L. J. Flores, and R. J. Reiter, "One molecule, many derivatives: a never-ending interaction of melatonin with reactive oxygen and nitrogen species?" Journal of Pineal Research, vol. 42, no. 1, pp. 28-42, 2007.

[19] L.-F. Ye, Z.-Z. Tao, Q.-Q. Hua et al., "Protective effect of melatonin against gentamicin ototoxicity," The Journal of Laryngology \& Otology, vol. 123, no. 6, pp. 598-602, 2009.

[20] C. Rodriguez, J. C. Mayo, R. M. Sainz et al., "Regulation of antioxidant enzymes: a significant role for melatonin," Journal of Pineal Research, vol. 36, no. 1, pp. 1-9, 2004.

[21] J. Espino, J. A. Pariente, and A. B. Rodríguez, "Oxidative stress and immunosenescence: therapeutic effects of melatonin," Oxidative Medicine and Cellular Longevity, vol. 2012, Article ID 670294, 9 pages, 2012.

[22] M. Karasek, "Melatonin, human aging, and age-related diseases," Experimental Gerontology, vol. 39, no. 11-12, pp. 17231729,2004

[23] R. J. Reiter, D. Tan, J. C. Mayo, R. M. Sainz, J. Leon, and Z. Czarnocki, "Melatonin as an antioxidant: biochemical mechanisms and pathophysiological implications in humans," Acta Biochimica Polonica, vol. 50, no. 4, pp. 1129-1146, 2003.

[24] L. Lin, Q.-X. Huang, S.-S. Yang, J. Chu, J.-Z. Wang, and Q. Tian, "Melatonin in Alzheimer's disease," International Journal of Molecular Sciences, vol. 14, no. 7, pp. 14575-14593, 2013.

[25] J. M. Olcese, C. Cao, T. Mori et al., "Protection against cognitive deficits and markers of neurodegeneration by long-term oral administration of melatonin in a transgenic model of Alzheimer disease," Journal of Pineal Research, vol. 47, no. 1, pp. 82-96, 2009.

[26] J. Z. Wang and Z. F. Wang, "Role of melatonin in Alzheimer-like neurodegeneration," Acta Pharmacologica Sinica, vol. 27, no. 1, pp. 41-49, 2006.

[27] V. Srinivasan, D. P. Cardinali, U. S. Srinivasan et al., "Therapeutic potential of melatonin and its analogs in Parkinson's disease: focus on sleep and neuroprotection," Therapeutic Advances in Neurological Disorders, vol. 4, no. 5, pp. 297-317, 2011. 
[28] L. Lin, Y. Du, S. Yuan, J. Shen, X. Lin, and Z. Zheng, "Serum melatonin is an alternative index of Parkinson's disease severity," Brain Research, vol. 1547, pp. 43-48, 2014.

[29] G. Rocchitta, R. Migheli, G. Esposito et al., "Endogenous melatonin protects L-DOPA from autoxidation in the striatal extracellular compartment of the freely moving rat: potential implication for long-term L-DOPA therapy in Parkinson's disease," Journal of Pineal Research, vol. 40, no. 3, pp. 204-213, 2006.

[30] E. Gitto, M. Karbownik, R. J. Reiter et al., "Effects of melatonin treatment in septic newborns," Pediatric Research, vol. 50, no. 6, pp. 756-760, 2001.

[31] A. M. Sánchez-Sánchez, V. Martín, G. García-Santos et al., "Intracellular redox state as determinant for melatonin antiproliferative vs cytotoxic effects in cancer cells," Free Radical Research, vol. 45, no. 11-12, pp. 1333-1341, 2011.

[32] R. M. Sainz, J. C. Mayo, C. Rodriguez, D. X. Tan, S. LopezBurillo, and R. J. Reiter, "Melatonin and cell death: differential actions on apoptosis in normal and cancer cells," Cellular and Molecular Life Sciences, vol. 60, no. 7, pp. 1407-1426, 2003.

[33] S. Cos, A. González, C. Martínez-Campa, M. D. Mediavilla, C. Alonso-González, and E. J. Sánchez-Barceló, "Estrogensignaling pathway: a link between breast cancer and melatonin oncostatic actions," Cancer Detection and Prevention, vol. 30, no. 2, pp. 118-128, 2006.

[34] A. N. Viswanathan and E. S. Schernhammer, "Circulating melatonin and the risk of breast and endometrial cancer in women," Cancer Letters, vol. 281, no. 1, pp. 1-7, 2009.

[35] S. M. Hill, T. Frasch, and T. Duplessis, "Molecular mechanisms of melatonin anticancer effects," Integrative Cancer Therapies, vol. 8, no. 4, pp. 337-346, 2009.

[36] A. Korkmaz, E. J. Sanchez-Barcelo, D. X. Tan, and R. J. Reiter, "Role of melatonin in the epigenetic regulation of breast cancer," Breast Cancer Research and Treatment, vol. 115, no. 1, pp. 13-27, 2009.

[37] N. Song, A. J. Kim, H. Kim et al., "Melatonin suppresses doxorubicin-induced premature senescence of A549 lung cancer cells by ameliorating mitochondrial dysfunction," Journal of Pineal Research, vol. 53, no. 4, pp. 335-343, 2012.

[38] P. Lissoni, M. Chilelli, S. Villa, L. Cerizza, and G. Tancini, "Five years survival in metastatic non-small cell lung cancer patients treated with chemotherapy alone or chemotherapy and melatonin: a randomized trial," Journal of Pineal Research, vol. 35, no. 1, pp. 12-15, 2003.

[39] S. Casado-Zapico, V. Martín, G. García-Santos et al., "Regulation of the expression of death receptors and their ligands by melatonin in haematological cancer cell lines and in leukaemia cells from patients," Journal of Pineal Research, vol. 50, no. 3, pp. 345-355, 2011.

[40] I. Bejarano, P. C. Redondo, J. Espino et al., "Melatonin induces mitochondrial-mediated apoptosis in human myeloid HL-60 cells," Journal of Pineal Research, vol. 46, no. 4, pp. 392-400, 2009.

[41] O. Trubiani, R. Recchioni, F. Moroni, J. Pizzicannella, S. Caputi, and R. di Primio, "Melatonin provokes cell death in human Blymphoma cells by mitochondrial-dependent apoptotic pathway activation," Journal of Pineal Research, vol. 39, no. 4, pp. 425-431, 2005.

[42] S. Rubio, F. Estévez, J. Cabrera, R. J. Reiter, J. Loro, and J. Quintana, "Inhibition of proliferation and induction of apoptosis by melatonin in human myeloid HL-60 cells," Journal of Pineal Research, vol. 42, no. 2, pp. 131-138, 2007.
[43] G. García-Santos, I. Antolín, F. Herrera, V. Martín, J. RodriguezBlanco, and M. del Pilar Carrera, "Melatonin induces apoptosis in human neuroblastoma cancer cells," Journal of Pineal Research, vol. 41, no. 2, pp. 130-135, 2006.

[44] V. Martín, G. García-Santos, J. Rodriguez-Blanco et al., "Melatonin sensitizes human malignant glioma cells against TRAILinduced cell death," Cancer Letters, vol. 287, no. 2, pp. 216-223, 2010.

[45] V. Martín, F. Herrera, P. Carrera-Gonzalez et al., "Intracellular signaling pathways involved in the cell growth inhibition of glioma cells by melatonin," Cancer Research, vol. 66, no. 2, pp. 1081-1088, 2006.

[46] J. Wang, H. Hao, L. Yao et al., "Melatonin suppresses migration and invasion via inhibition of oxidative stress pathway in glioma cells," Journal of Pineal Research, vol. 53, no. 2, pp. 180-187, 2012.

[47] S. Casado-Zapico, J. Rodriguez-Blanco, G. García-Santos et al., "Synergistic antitumor effect of melatonin with several chemotherapeutic drugs on human Ewing sarcoma cancer cells: potentiation of the extrinsic apoptotic pathway," Journal of Pineal Research, vol. 48, no. 1, pp. 72-80, 2014.

[48] G. García-Santos, V. Martin, J. Rodríguez-Blanco et al., "Fas/Fas ligand regulation mediates cell death in human Ewings sarcoma cells treated with melatonin," British Journal of Cancer, vol. 106, no. 7, pp. 1288-1296, 2012.

[49] B. H. Ali and M. S. Al Moundhri, "Agents ameliorating or augmenting the nephrotoxicity of cisplatin and other platinum compounds: a review of some recent research," Food and Chemical Toxicology, vol. 44, no. 8, pp. 1173-1183, 2006.

[50] M. Hara, M. Yoshida, H. Nishijima et al., "Melatonin, a pineal secretory product with antioxidant properties, protects against cisplatin-induced nephrotoxicity in rats," Journal of Pineal Research, vol. 30, no. 3, pp. 129-138, 2001.

[51] G. Sener, L. Kabasakal, S. Arbakb, S. Onerb, F. Ercanb, and M. K. Uysal, "The protective effect of melatonin on cisplatin nephrotoxicity," Fundamental \& Clinical Pharmacology, vol. 14, pp. 553-560, 2000.

[52] U. Kilic, E. Kilic, Z. Tuzcu et al., "Melatonin suppresses cisplatininduced nephrotoxicity via activation of Nrf-2/HO-1 pathway," Nutrition \& Metabolism, vol. 10, no. 1, article 7, 2013.

[53] H. Parlakpinar, E. Sahna, M. K. Ozer, F. Ozugurlu, N. Vardi, and A. Acet, "Physiological and pharmacological concentrations of melatonin protect against cisplatin-induced acute renal injury," Journal of Pineal Research, vol. 33, no. 3, pp. 161-166, 2002.

[54] K. E. Atalik, B. Keleş, Y. Uyar, M. A. Dündar, M. Oz, and H. H. Esen, "Vasoprotection by melatonin and quercetin in rats treated with cisplatin," Indian Journal of Experimental Biology, vol. 48, no. 12, pp. 1188-1193, 2010.

[55] Y. O. Ilbey, E. Ozbek, A. Simsek, A. Otunctemur, M. Cekmen, and A. Somay, "Potential chemoprotective effect of melatonin in cyclophosphamide- and cisplatin-induced testicular damage in rats," Fertility and Sterility, vol. 92, no. 3, pp. 1124-1132, 2009.

[56] A. Ateşşahin, E. Sahna, G. Türk et al., "Chemoprotective effect of melatonin against cisplatin-induced testicular toxicity in rats," Journal of Pineal Research, vol. 41, no. 1, pp. 21-27, 2006.

[57] M. A. Lopez-Gonzalez, J. M. Guerrero, R. Torronteras, C. Osuna, and F. Delgado, "Ototoxicity caused by aminoglycosides is ameliorated by melatonin without interfering with the antibiotic capacity of the drugs," Journal of Pineal Research, vol. 28, no. 1, pp. $26-33,2000$.

[58] O. W. Guthrie, "Aminoglycoside induced ototoxicity," Toxicology, vol. 249, no. 2-3, pp. 91-96, 2008. 
[59] J.-B. Kim, J. Y. Jung, J.-C. Ahn, C. K. Rhee, and H. Hwang, "Antioxidant and anti-apoptotic effect of melatonin on the vestibular hair cells of rat utricles," Clinical and Experimental Otorhinolaryngology, vol. 2, no. 1, pp. 6-12, 2009.

[60] E. Bas, T. R. Van De Water, C. Gupta et al., "Efficacy of three drugs for protecting against gentamicin-induced hair cell and hearing losses," British Journal of Pharmacology, vol. 166, no. 6, pp. 1888-1904, 1888.

[61] T. Erdem, O. Ozturan, M. Iraz, M. C. Miman, and E. Olmez, "Dose-dependent dual effect of melatonin on ototoxicity induced by amikacin in adult rats," European Archives of OtoRhino-Laryngology, vol. 262, no. 4, pp. 314-321, 2005.

[62] J. G. Kalkanis, C. Whitworth, and L. P. Rybak, "Vitamin E reduces cisplatin ototoxicity," Laryngoscope, vol. 114, no. 3, pp. 538$542,2004$.

[63] S. A. Tokgöz, E. Vuralkan, N. D. Sonbay et al., "Protective effects of vitamins $\mathrm{E}, \mathrm{B}$ and $\mathrm{C}$ and $\mathrm{L}$-carnitine in the prevention of cisplatin-induced ototoxicity in rats," The Journal of Laryngology \& Otology, vol. 126, no. 5, pp. 464-469, 2012.

[64] G. Simşek, S. A. Tokgoz, E. Vuralkan, M. Caliskan, O. Besalti, and I. Akin, "Protective effects of resveratrol on cisplatindependent inner-ear damage in rats," European Archives of OtoRhino-Laryngology, vol. 270, no. 6, pp. 1789-1793, 2013.

[65] T. Erdem, T. Bayindir, A. Filiz, M. Iraz, and E. Selimoglu, "The effect of resveratrol on the prevention of cisplatin ototoxicity," European Archives of Oto-Rhino-Laryngology, vol. 269, no. 10, pp. 2185-2188, 2012.

[66] A. L. L. Sampaio, M. A. Dias, A. R. Venosa, P. L. Tauil, E. Alencar, and C. A. C. P. Oliveira, "The Effect of Gingko Biloba (EGB 761) as an otoprotector against cisplatin ototoxicity," Otolaryngology-Head and Neck Surgery, vol. 149, supplement 2, pp. P222-P223, 2013.

[67] B. A. Abdel-Wahab and S. M. Abd El-Aziz, "Ginkgo biloba protects against intermittent hypoxia-induced memory deficits and hippocampal DNA damage in rats," Phytomedicine, vol. 19, no. 5, pp. 444-450, 2012.

[68] X. Huang, C. A. Whitworth, and L. P. Rybak, "Ginkgo Biloba extract (EGb 761) protects against cisplatin-induced ototoxicity in rats," Otology \& Neurotology, vol. 28, no. 6, pp. 828-833, 2007.

[69] S. J. Choi, S. W. Kim, J. B. Lee et al., "Gingko biloba extracts protect auditory hair cells from cisplatin-induced ototoxicity by inhibiting perturbation of gap junctional intercellular communication," Neuroscience, vol. 244, pp. 49-61, 2013.

[70] M. A. Hyppolito, M. Rossato, and F. Holanda, "Ototoxicidade da cisplatina e otoproteção pelo extrato de ginkgo biloba às células ciliadas externas: estudo anatômico e eletrofisiológico," Brazilian Journal of Otorhinolaryngology, vol. 69, no. 4, pp. 504511, 2003.

[71] C. J. Tian, Y. J. Kim, S. W. Kim, H. J. Lim, Y. S. Kim, and Y.H. Choung, "A combination of cilostazol and Ginkgo biloba extract protects against cisplatin-induced Cochleo-vestibular dysfunction by inhibiting the mitochondrial apoptotic and ERK pathways," Cell Death and Disease, vol. 4, no. 2, p. e509, 2013.

[72] S. J. Hong, G. J. Im, J. Chang et al., "Protective effects of edaravone against cisplatin-induced hair cell damage in zebrafish," International Journal of Pediatric Otorhinolaryngology, vol. 77, no. 6, pp. 1025-1031, 2013.

[73] O. Kuduban, C. Kucur, E. Sener, H. Suleyman, and F. Akcay, "The role of thiamine pyrophosphate in prevention of cisplatin ototoxicity in an animal model," The Scientific World Journal, vol. 2013, Article ID 182694, 5 pages, 2013.
[74] G. Lorito, S. Hatzopoulos, G. Laurell et al., "Dose-dependent protection on cisplatin-induced ototoxicity - an electrophysiological study on the effect of three antioxidants in the SpragueDawley rat animal model," Medical Science Monitor, vol. 17, no. 8, pp. BR179-BR186, 2011.

[75] K. C. M. Campbell, R. P. Meech, L. P. Rybak, and L. F. Hughes, "The effect of D-methionine on cochlear oxidative state with and without cisplatin administration: mechanisms of otoprotection," Journal of the American Academy of Audiology, vol. 14, no. 3, pp. 144-156, 2003.

[76] K. D. Korver, L. P. Rybak, C. Whitworth, and K. M. Campbell, "Round window application of D-methionine provides complete cisplatin otoprotection," Otolaryngology-Head and Neck Surgery, vol. 126, no. 6, pp. 683-689, 2002.

[77] M. G. Riga, L. Chelis, S. Kakolyris et al., "Transtympanic injections of $\mathrm{N}$-acetylcysteine for the prevention of cisplatin-induced ototoxicity: a feasible method with promising efficacy," The American Journal of Clinical Oncology: Cancer Clinical Trials, vol. 36, no. 1, pp. 1-6, 2013.

[78] H. J. Jeong, Y. Choi, M. H. Kim et al., "Rosmarinic acid, active component of dansam-eum attenuates ototoxicity of cochlear hair cells through blockage of caspase-1 activity," PLOS ONE, vol. 6, no. 4, Article ID e18815, 2011.

[79] S. Celebi, M. M. Gurdal, M. H. Ozkul, H. Yasar, and H. H. Balikci, "The effect of intratympanic vitamin $\mathrm{C}$ administration on cisplatin-induced ototoxicity," European Archives of OtoRhino-Laryngology, vol. 270, no. 4, pp. 1293-1297, 2013.

[80] M. Paksoy, E. Ayduran, A. Sanl, M. Eken, S. Aydin, and Z. A. Oktay, "The protective effects of intratympanic dexamethasone and vitamin $\mathrm{E}$ on cisplatin-induced ototoxicity are demonstrated in rats," Medical Oncology, vol. 28, no. 2, pp. 615-621, 2011.

[81] D. Murphy and S. J. Daniel, "Intratympanic dexamethasone to prevent cisplatin ototoxicity: a guinea pig model," Otolaryngology: Head and Neck Surgery, vol. 145, no. 3, pp. 452-457, 2011.

[82] A. G. Shafik, R. H. Elkabarity, M. T. Thabet, N. B. Soliman, and N. K. Kalleny, "Effect of intratympanic dexamethasone administration on cisplatin-induced ototoxicity in adult guinea pigs," Auris Nasus Larynx, vol. 40, no. 1, pp. 51-60, 2013.

[83] G. W. Hill, D. K. Morest, and K. Parham, "Cisplatin-induced ototoxicity: effect of intratympanic dexamethasone injections," Otology \& Neurotology, vol. 29, no. 7, pp. 1005-1011, 2008. 


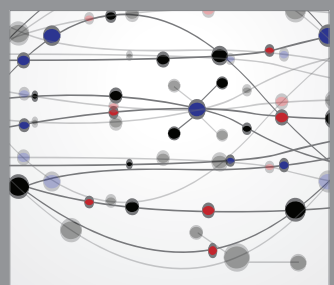

The Scientific World Journal
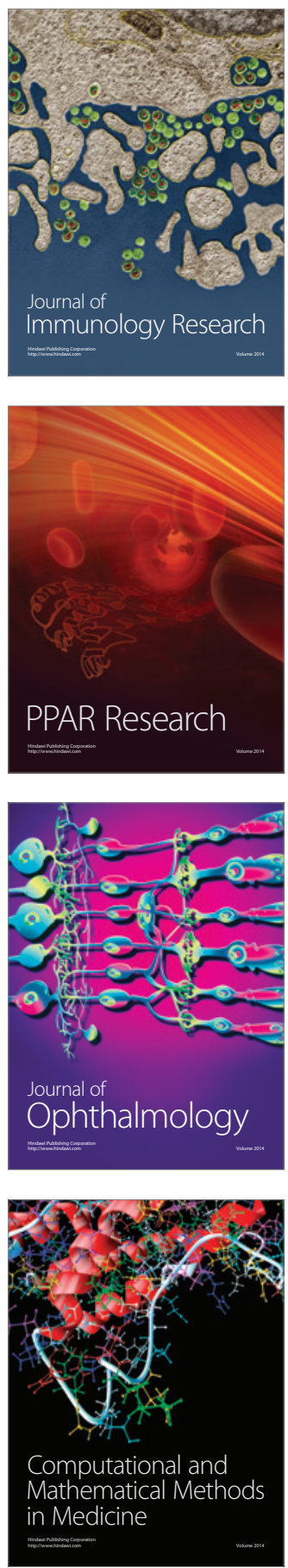

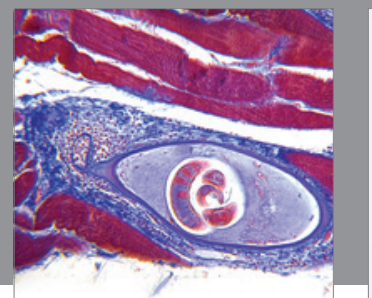

Gastroenterology

Research and Practice
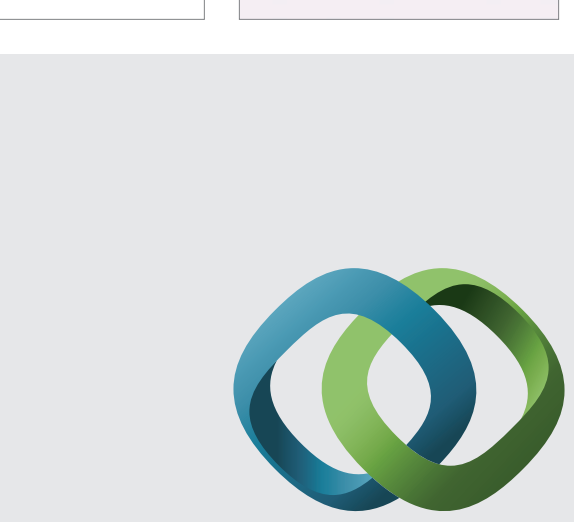

\section{Hindawi}

Submit your manuscripts at

http://www.hindawi.com
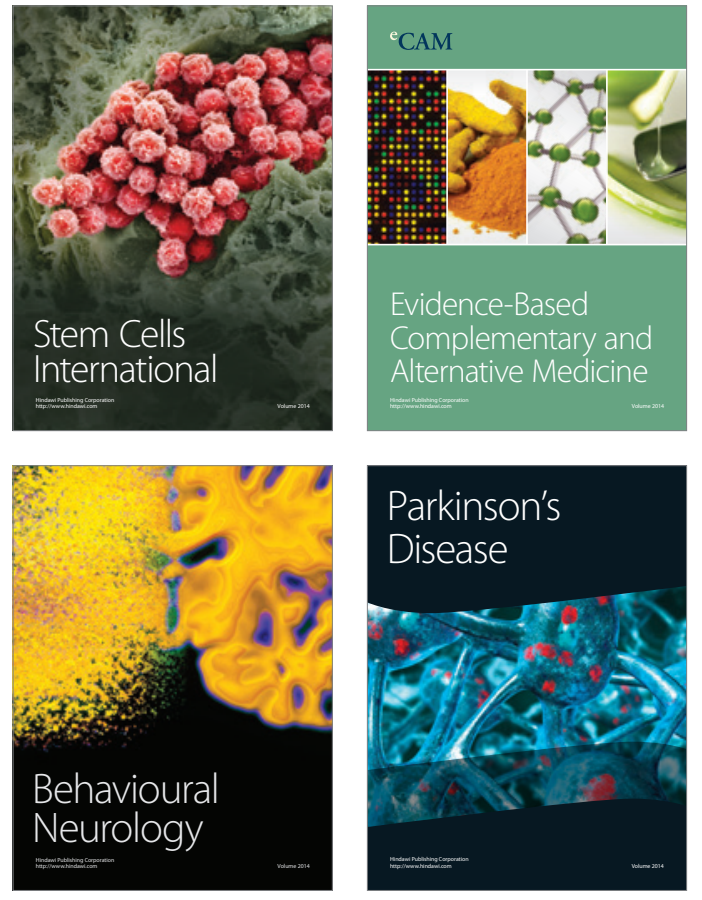
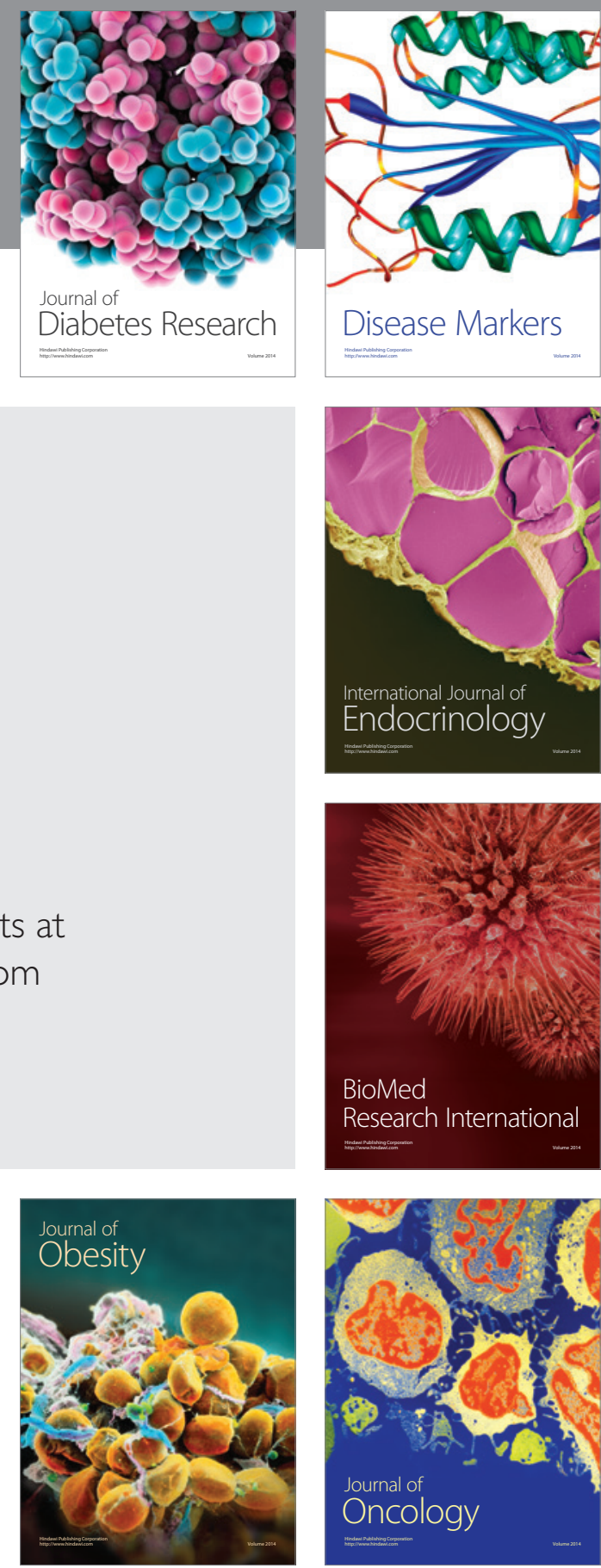

Disease Markers
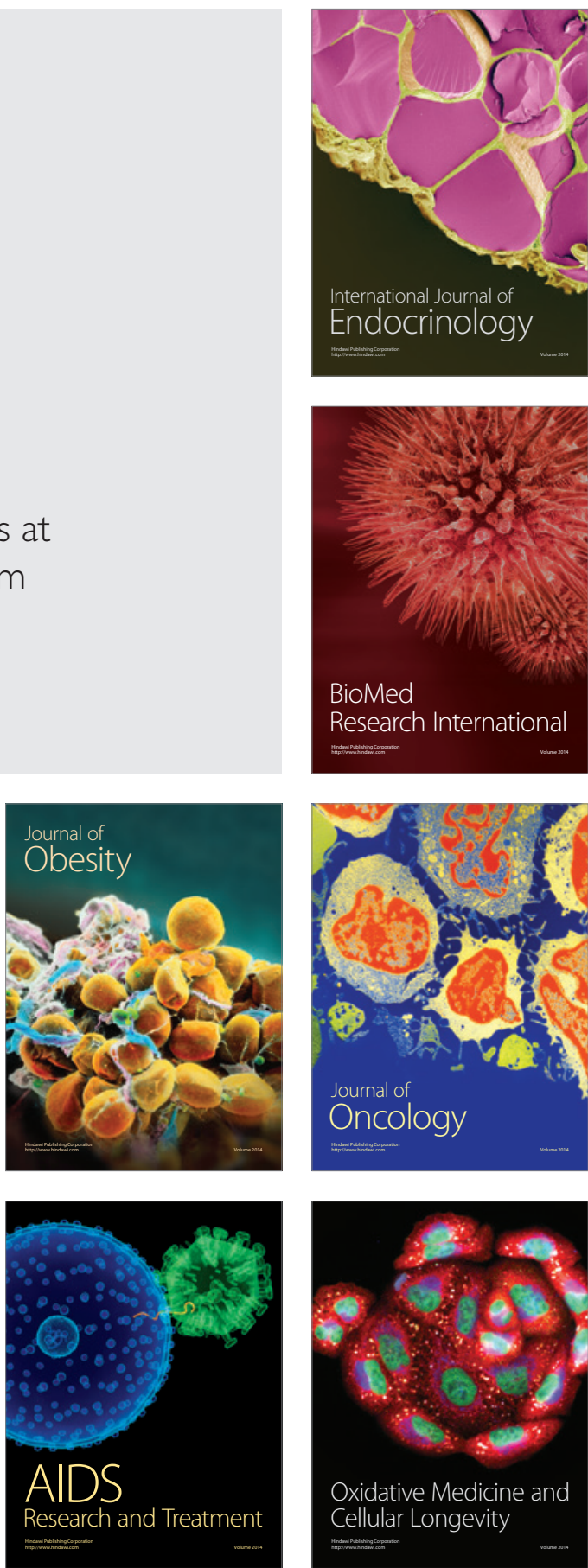